\title{
Acute Focal Bacterial Nephritis Associated With Reversible Splenial Corpus Callosum Lesion
}

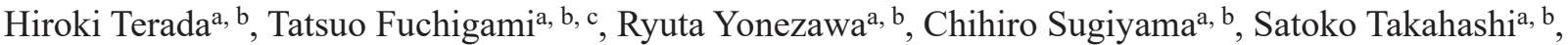 \\ Yuko Moriuchi ${ }^{\mathrm{a}, \mathrm{b}}$, Tetsuharu Kamioka ${ }^{\mathrm{a}}$, Yuichi Takahashi ${ }^{\mathrm{a}}$, Miho Yamanishia, Mina Otsubo ${ }^{\mathrm{a}}$, \\ Yuko Ohashi ${ }^{\mathrm{a}}$, Jun Negishi ${ }^{\mathrm{a}}$, Ichiro Morioka ${ }^{\mathrm{b}}$
}

\begin{abstract}
Acute focal bacterial nephritis (AFBN) is a localized bacterial infection of the kidney, which presents as an inflammatory mass without formation of a frank abscess. Rare cases of AFBN are accompanied by neurological symptoms such as meningeal irritation, unconsciousness, and seizures. Clinically mild encephalitis/encephalopathy with a reversible splenial lesion (MERS) is characterized by transient high signal intensity splenial lesions on diffusion-weighted magnetic resonance imaging. MERS can be divided into two types: type 1 with an isolated splenium of the corpus callosum and type 2 with extensive white matter and/ or entire callosal lesions. We experienced three rare cases of pediatric AFBN associated with neurological symptoms, including unconsciousness and reversible splenial corpus callosum lesion. The first case was an 8-year-old girl who had neurological symptoms, including unconsciousness, and AFBN was associated with MERS type 2. The second (5-year-old boy) and third (5-year-old girl) cases had neurological symptoms, including unconsciousness and AFBN with MERS type 1. Two of the three cases had AFBN caused by Escherichia coli. All three cases showed high levels of urinary $\beta 2$-microglobulin (B2MG). AFBN should be suspected in children with fever, rapid clinical deterioration, neurological symptoms including unconsciousness, and high urinary B2MG level. We recommend that abdominal enhanced computed tomography should be performed for the diagnosis of AFBN.
\end{abstract}

Keywords: Acute focal bacterial nephritis; MERS; Neurological symptoms; Unconsciousness; Urinary $\beta 2$-microglobulin; Escherichia coli; Abdominal enhanced CT; Brain MRI

\section{Introduction}

Acute focal bacterial nephritis (AFBN) is a localized bacte-

Manuscript submitted June 25, 2020, accepted July 9, 2020

Published online July 30, 2020

${ }^{a}$ Department of Pediatrics, IMS Fujimi General Hospital, Fujimi, Japan bepartment of Pediatrics and Child Health, Nihon University School of Medicine, Tokyo, Japan

${ }^{c}$ Corresponding Author: Tatsuo Fuchigami, Department of Pediatrics, IMS Fujimi General Hospital, 1967-1, Tsuruma, Fujimi-shi, Saitama 354-0021, Japan. Email: tfuchigami@ims.gr.jp

doi: https://doi.org/10.14740/ijcp395 rial infection of the kidney, which presents as an inflammatory mass without the formation of a frank abscess. It was first described in adults by Rosenfield et al in 1979 and was previously referred to as lobar nephronia [1]. AFBN is considered the midpoint in the spectrum of upper urinary tract infections, ranging from pyelonephritis to intrarenal abscesses, and might represent a relatively early stage of renal abscesses [2]. Most children with AFBN exhibit nonspecific symptoms such as fever, vomiting, and abdominal pain. In addition, pyuria and bacteriuria commonly associated with urinary tract infections are often absent in these cases. Therefore, they are diagnosed as fever of unknown origin [3,4]. Rare cases of AFBN are accompanied by neurological symptoms such as meningeal irritation, unconsciousness, and seizures [5-7].

Tada et al [8] identified clinically mild encephalitis/encephalopathy with a reversible splenic lesion (MERS). It is characterized by transient high signal intensity splenial lesions on diffusion-weighted magnetic resonance imaging (DWMRI). MERS is a new type of acute encephalopathy that might sometimes be accompanied by symmetrical white matter lesions with a mild clinical course and has a good outcome.

We experienced three rare pediatric cases of AFBN associated with neurological symptoms including unconsciousness and reversible splenial corpus callosum lesion.

\section{Case Reports}

\section{Case 1}

An 8-year-old girl had fever and vomiting since 1 day before hospitalization. On the day of admission, she had abnormal speech and visited a local clinic, and was then transferred to our hospital. She had no remarkable family, birth or developmental, and vaccination history. She also had an overactive bladder. At admission, she was $139.0 \mathrm{~cm}$ in height and weighed $40.6 \mathrm{~kg}$. She had a body temperature of $40.6^{\circ} \mathrm{C}$, heart rate of 128 beats $/ \mathrm{min}$, blood pressure of $107 / 56 \mathrm{~mm} \mathrm{Hg}$, and percutaneous oxygen saturation of $99 \%$. Her general condition was poor and confused (Glasgow Coma Scale score of 14 (E4, V4, M6)). There was no neck stiffness, and the chest and abdominal examination revealed no abnormal findings. There was pain over the costovertebral angle on the left side. Laboratory findings on admission were white blood cell count (WBC) 


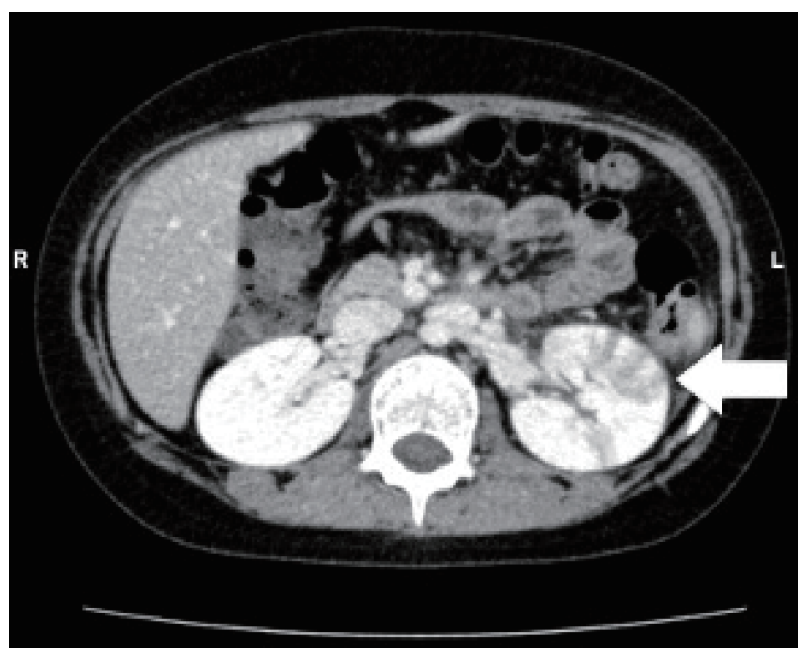

Figure 1. Abdominal enhanced computed tomography (CT) shows enlargement of the left kidney, with multiple wedge-shaped defects (arrow).

23,600/ $\mu \mathrm{L}$, hemoglobin $(\mathrm{Hb}) 13.6 \mathrm{~g} / \mathrm{dL}$, platelet (Plt) 260,000 $/ \mu \mathrm{L}$, aspartate aminotransferase (AST) $29 \mathrm{U} / \mathrm{L}$, alanine aminotransferase (ALT) $26 \mathrm{U} / \mathrm{L}$, urea nitrogen (UN) $10.9 \mathrm{mg} / \mathrm{dL}$, creatinine (Cre) $0.43 \mathrm{mg} / \mathrm{dL}, \mathrm{C}$-reactive protein (CRP) 24.24 $\mathrm{mg} / \mathrm{dL}, \mathrm{Na} 136 \mathrm{mEq} / \mathrm{L}, \mathrm{K} 3.4 \mathrm{mEq} / \mathrm{L}$, and Cl $98 \mathrm{mEq} / \mathrm{L}$. Midstream urinalysis was negative for protein and occult blood. However, it showed WBC 30 - 49 cells /high power field (HF), urinary $\beta 2$-microglobulin (B2MG) 6,433 $\mu \mathrm{g} / \mathrm{L}$ (normal: 0 - 289 $\mu \mathrm{g} / \mathrm{L}$ ) and $\mathrm{N}$-acetyl-beta-D-glucosaminidase (NAG) $3.2 \mathrm{IU} / \mathrm{L}$ (normal: 0 - 11.5 IU/L). Cerebrospinal fluid (CSF) analysis did not show any pleocytosis, elevated protein levels, or decrease in glucose levels. The patient was treated with intravenous 100 $\mathrm{mg} / \mathrm{kg}$ daily ampicillin hydrate (ABPC) and $50 \mathrm{mg} / \mathrm{kg}$ daily ceftriaxone sodium hydrate (CTRX) because the cause of fever was suspected to be urinary tract infection and/or sepsis. Blood and CSF cultures showed no pathogens. However, from catheter urine culture, the organism was identified as Escherichia coli $\left(E\right.$. coli) $10^{4}$ colony forming unit (CFU)/mL, and intravenous ABPC therapy was stopped after 6 days. Abdominal computed tomography (CT) and brain MRI were performed on the second day of admission. Abdominal enhanced CT showed enlargement of the left kidney, with multiple wedge- shaped defects (Fig. 1). Brain T1 and T2-weighted images did not reveal any structural abnormalities. DW-MRI showed a focal, high-intensity lesion in the splenium of the corpus callosum. Apparent diffusion coefficient (ADC) mapping showed decreased ADC values at the site of the abnormality, and DW image also showed bilateral symmetrical high-intensity white matter lesions at the frontal and parietal lobes (Fig. 2). These findings strongly suggested acute encephalopathy and AFBN with $E$. coli infection. On the third day of admission, her fever improved. On the 10th day after admission, she underwent repeat brain MRI, which showed that the lesion had disappeared. The final diagnosis was AFBN with MERS type 2. On the 14th day, she underwent voiding cystourethrography (VCUG), which revealed voiding dysfunction. After 14 days of CTRX therapy, the antibiotic was changed to oral administration of $40 \mathrm{mg} / \mathrm{kg}$ daily cefaclor for 7 days and she was discharged on the 15th day. After discharge, technetium 99m-dimercaptosuccinic acid (99mTc-DMSA) scintigraphy was performed and revealed no abnormal findings. She was managed without surgery and continued medication for overactive bladder. Subsequently, she had a good condition and no remarkable change.

\section{Case 2}

A 5-year-old boy developed fever and cough a day before admission. He visited a local clinic and was diagnosed with acute upper respiratory tract inflammation. However, his fever and cough continued, and the chest radiograph showed abnormal shadows indicative of pneumonia. He also showed abnormal behavior and disorder of consciousness the next day and was transferred to our hospital for suspected acute pneumonia. He had no remarkable family, birth or developmental, and vaccination history. On examination at admission, he was 118.5 $\mathrm{cm}$ in height and weighed $22.3 \mathrm{~kg}$. He had a body temperature of $40.2^{\circ} \mathrm{C}$, heart rate of 156 beats/min, and percutaneous oxygen saturation of $98 \%$. His general condition was poor, and he was confused (Glasgow coma scale score of 14 (E4, V4, M6)). There was no neck stiffness. There were no abnormal findings on chest and abdominal examination. There was no costovertebral angle pain and tenderness. Laboratory findings on admission were WBC 22,110/ $\mathrm{LL}$, Hb $13.5 \mathrm{~g} / \mathrm{dL}$, Plt 200,000 / $\mu \mathrm{L}$, AST $30 \mathrm{U} / \mathrm{L}$, ALT $12 \mathrm{U} / \mathrm{L}, \mathrm{UN} 12.6 \mathrm{mg} / \mathrm{dL}$, Cre $0.46 \mathrm{mg} /$
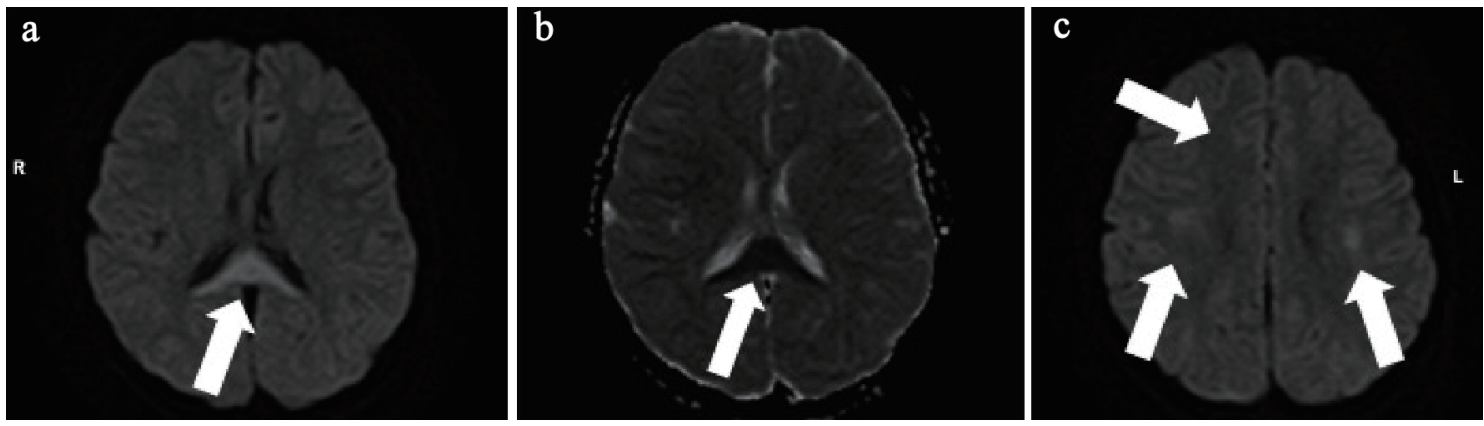

Figure 2. (a) Brain diffusion-weighted (DW)-MRI shows focal, high-intensity lesion in the splenium of the corpus callosum (arrow). (b) Apparent diffusion coefficient (ADC) mapping shows decreased ADC values at the region of the abnormality (arrow). (c) DW-MRI shows bilateral symmetrical high intensity white matter lesions at the frontal and parietal lobes (arrows). 

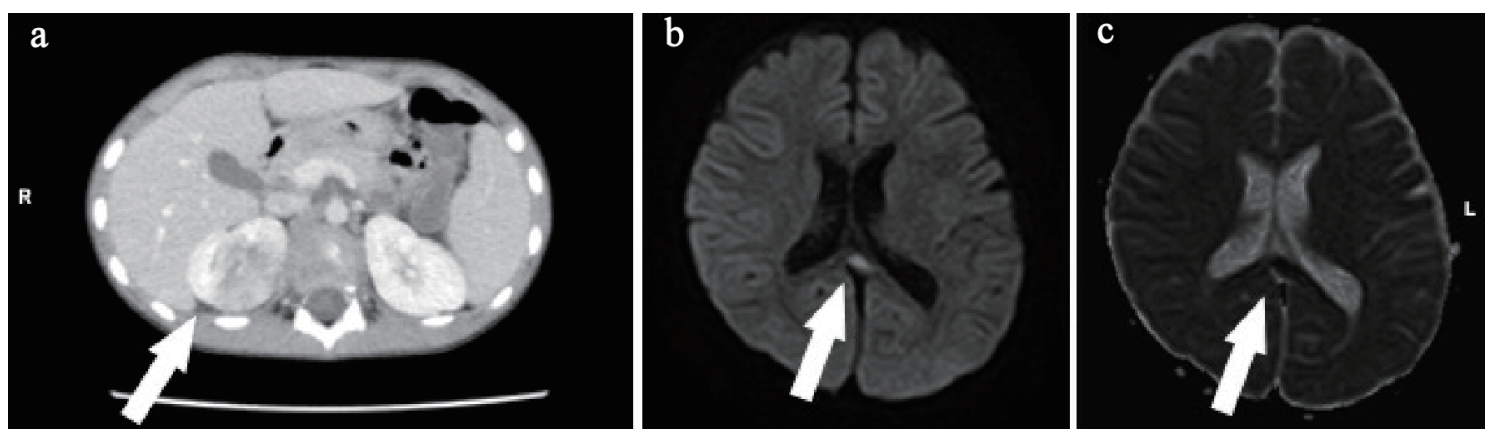

Figure 3. (a) Abdominal enhanced computed tomography (CT) shows right kidney with multiple wedge-shaped defects (arrow). (b) Brain diffusion-weighted magnetic resonance imaging (DW-MRI) shows a focal, high-intensity lesion in the splenium of the corpus callosum (arrow). (c) Apparent diffusion coefficient (ADC) mapping shows decreased ADC values at the region of the abnormality (arrows).

dL, CRP $9.98 \mathrm{mg} / \mathrm{dL}, \mathrm{Na} 132 \mathrm{mEq} / \mathrm{L}, \mathrm{K} 3.5 \mathrm{mEq} / \mathrm{L}$, and $\mathrm{Cl}$ $95 \mathrm{mEq} / \mathrm{L}$. Catheter urinalysis was negative for protein and occult blood. Urinary WBC showed 20 - 29 cells/HF, B2MG was $912 \mu \mathrm{g} / \mathrm{L}$, and NAG was $7.4 \mathrm{IU} / \mathrm{L}$. Blood and urine cultures showed no pathogens. On the day of admission, these findings were suggestive of acute pneumonia and urinary tract infection. The patient was treated with intravenous $150 \mathrm{mg} / \mathrm{kg}$ daily ABPC and $100 \mathrm{mg} / \mathrm{kg}$ daily CTRX. However, his fever and abnormal speech with confusion continued. We suspected AFBN and MERS because of the findings of urinary WBC count and high level of B2MG; hence, abdominal CT and brain MRI were performed on the third day of admission. Abdominal enhanced CT showed multiple wedge-shaped defects in the right kidney (Fig. 3a). The diagnosis was confirmed as AFBN. Brain DW-MRI showed high intensity in the splenium of the corpus callosum (Fig. 3b, c). On the fourth day of admission, his fever improved. On the 12th day after admission, he underwent repeat brain MRI, which showed that the lesion had disappeared. The final diagnosis was AFBN with MERS type 1. On the 13th day after admission, he underwent VCUG, which revealed grade 3 vesicoureteral reflux (VUR) on the right side. After 14 days of ABPC and CTRX, the therapy was changed to oral administration of $30 \mathrm{mg} / \mathrm{kg}$ daily cefaclor for 7 days, and he was discharged on the 15 th day. After discharge, $99 \mathrm{mTc}-$ DMSA scintigraphy was performed, which revealed right renal dysfunction as left renal uptake rate of $22.2 \%$ and right renal uptake rate of $17.3 \%$ (normal uptake rate: $>20 \%$ ). Preventive surgery for the management of VUR was performed 6 months after discharge. Subsequently, he had a good condition and no remarkable change.

\section{Case 3}

A 5-year-old girl developed fever, abdominal pain, and vomiting since morning on the day of admission, and visited a local clinic. At the clinic, she developed a disorder of consciousness and was transferred to our hospital. She had no remarkable family, birth or developmental, and vaccination history. She had chronic constipation and had been hospitalized for left-sided AFBN 6 months ago. Her VCUG showed no abnormal findings. At admission, she was $106.5 \mathrm{~cm}$ in height and weighed
$18.6 \mathrm{~kg}$. She had a body temperature of $39.0^{\circ} \mathrm{C}$, heart rate of 164 beats/min, and percutaneous oxygen saturation of $99 \%$. Her general condition was poor, and she had somnolence with a Glasgow Coma Scale score of E4, V1, M6. There was no neck stiffness. There were no abnormal findings on the chest and abdominal examination. There was no pain or tenderness over the costovertebral angle. Laboratory findings on admission were

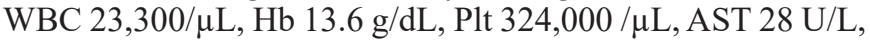
ALT $14 \mathrm{U} / \mathrm{L}, \mathrm{UN} 14.9 \mathrm{mg} / \mathrm{dL}$, Cre $0.30 \mathrm{mg} / \mathrm{dL}$, CRP $0.82 \mathrm{mg} /$ $\mathrm{dL}, \mathrm{Na} 135 \mathrm{mEq} / \mathrm{L}, \mathrm{K} 3.8 \mathrm{mEq} / \mathrm{L}$, and Cl $100 \mathrm{mEq} / \mathrm{L}$. Catheter urinalysis was negative for protein and occult blood. Urinary WBC showed 1 - 4 cells/HF. Urinary B2MG was 1,527 $\mu \mathrm{g} / \mathrm{L}$, and NAG was $3.7 \mathrm{IU} / \mathrm{L}$. The patient was treated with intravenous $100 \mathrm{mg} / \mathrm{kg}$ daily cefotaxime sodium (CTX) because the origin of her fever was suspected to be urinary tract infection. Blood culture showed no pathogens. However, the organism was identified as E. coli $10^{5} \mathrm{CFU} / \mathrm{mL}$ from the catheter urine culture. Abdominal CT and brain MRI were performed on the second day of admission. Abdominal enhanced CT showed that the left kidney had multiple wedge-shaped defects (Fig. 4a). DW-MRI showed a focal, high-intensity lesion in the splenium of the corpus callosum. ADC mapping showed decreased $\mathrm{ADC}$ values in the area of the abnormality (Fig. 4b, c). The final diagnosis was AFBN with MERS type 1. On the third day of admission, her consciousness improved and fever improved on the sixth day. After 14 days of CTX, the therapy was changed to oral administration of $30 \mathrm{mg} / \mathrm{kg}$ daily cefaclor for 7 days and she was discharged on the 15th day. After discharge, VCUG and 99mTc-DMSA scintigraphy were performed. Her VCUG revealed grade 2 VUR on the right and occult VUR at the left kidney. 99mTc-DMSA scintigraphy showed no abnormal findings. Surgery was performed for the prevention of VUR 6 months after discharge. Subsequently, she had a good condition and no remarkable change.

\section{Discussion}

We experienced three rare pediatric cases of AFBN associated with neurological symptoms, including unconsciousness and reversible splenial corpus callosum lesion.

Most children with AFBN exhibit nonspecific symptoms 

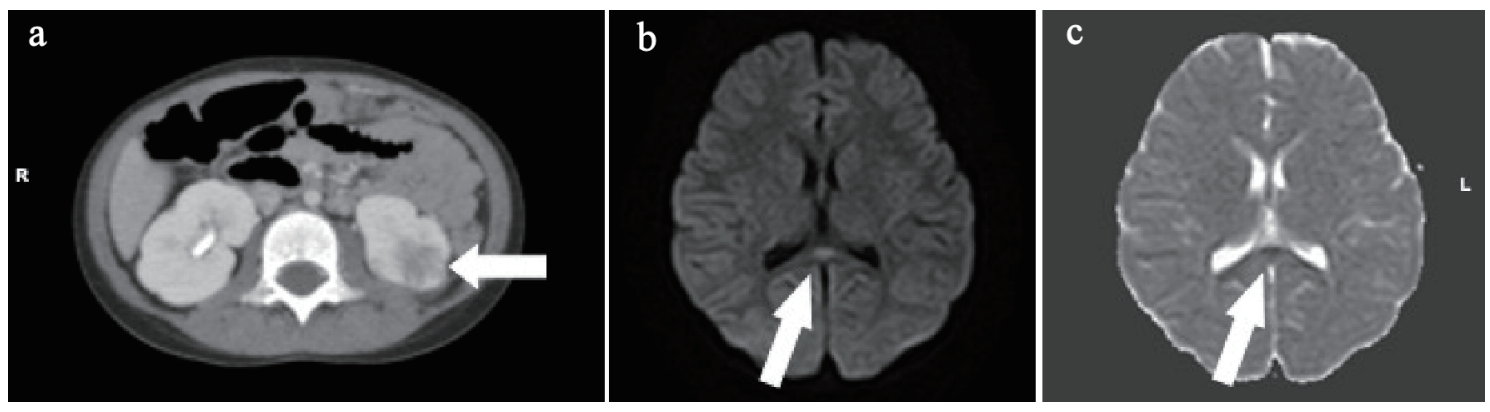

Figure 4. (a) Abdominal enhanced computed tomography (CT) shows the left kidney with multiple wedge-shaped defects (arrow). (b) Brain diffusion-weighted magnetic resonance imaging (DW-MRI) shows a focal, high-intensity lesion in the splenium of the corpus callosum (arrow). (c) Apparent diffusion coefficient (ADC) mapping shows decreased ADC values at the region of the abnormality (arrows).

such as fever, vomiting, and abdominal pain and did not show neurological symptoms. The most common clinical manifestations of patients with MERS are neurological disorders such as delirium, disorder of consciousness, and seizures. All our three cases related to AFBN with MERS showed neurological disorder, including disturbed consciousness.

Takahashi et al [9] suggested that MERS should be divided into two types: type 1 with an isolated splenium of the corpus callosum and type 2 with extensive white matter and/ or entire callosal lesions. Our first case had neurological symptoms, including unconsciousness, and AFBN was associated with MERS type 2. The second and third cases with neurological symptoms, including unconsciousness, had AFBN with MERS type 1.

A total of 983 cases reported had acute encephalopathy between 2007 and 2010 in Japan. Among 153 cases of MERS, the pathogens identified were influenza virus $(34.4 \%)$, rotavirus $(11.7 \%)$, mumps virus $(3.9 \%)$, herpesvirus $6(2.0 \%)$, and bacterial (3.3\%) infections [10]. Bacterial pathogens are rare, but Streptococcus and E. coli have been reported [11].

Cheng et al reported that $E$. coli was the most common pathogen cultured from 59 of 61 urine samples from 80 patients with AFBN [3]. Okamoto et al [12] reported MERS with a febrile urinary tract infection due to Klebsiella pneumoniae, and Kometani et al [6] reported two cases of MERS associated with AFBN caused by Enterococcus faecalis. Kasuga et al [7] reported a case of MERS with AFBN caused by E. coli. In this study, E. coli was identified in two of the three cases. Although several viruses and rarely bacteria are associated with MERS, the pathophysiological mechanisms are unclear. It has been suggested that MERS might be caused by intramyelinic axonal edema or local inflammatory cell infiltration [8]. Takanashi et al [13] reported that most patients with MERS have mild hyponatremia. Hypotonic hyponatremia results in the entry of water into the brain, resulting in central edema, headache, nausea, vomiting, confusion, and seizures. One patient also had a low serum sodium level ( $\mathrm{Na} 132 \mathrm{mEq} / \mathrm{L})$ on admission. Kometani et al [6] reported a significant elevation of serum and CSF interleukin-6 (IL-6) levels in MERS associated with AFBN. The elevated CSF levels of IL-6, which are remotely induced by localized inflammation in the kidney, might be related to the development of MERS.

Renal ultrasonography is the best screening and diagnos- tic method for the evaluation of renal inflammatory disease in the pediatric population. However, both false-positive and false-negative findings have been reported. CT with contrast is considered the gold standard for the diagnosis of AFBN and typically shows poorly enhanced solid wedge-shaped defects [14]. We also diagnosed AFBN using abdominal contrast CT.

Urinary B2MG and NAG are useful markers for the diagnosis of urinary tract infections [15]. Previous reports have also shown that urinary B2MG and/or NAG are useful markers for the diagnosis of AFBN with and without pyuria $[4,7,16]$. Therefore, we suspected AFBN based on the high levels of urinary B2MG. In this study, all three cases of AFBN with MERS showed high urinary B2MG levels; however, the urinary NAG was not elevated.

In conclusion, AFBN should be suspected in children with fever, rapid clinical deterioration, and neurological symptoms, including unconsciousness with high urinary B2MG levels. We recommend that abdominal enhanced CT should be performed to diagnose AFBN.

\section{Acknowledgments}

None to declare.

\section{Financial Disclosure}

The authors received no financial support for the research and/ or authorship of this article.

\section{Conflict of Interest}

The authors declare no potential conflict of interest with respect to the authorship and/or publication of this article.

\section{Informed Consent}

Informed written consents for publication of these cases were obtained from the patients' parents. 


\section{Author Contributions}

H. Terada, R. Yonezawa, C. Sugiyama, S. Takahashi, Y. Moriuchi, T. Kamioka, Y. Takahashi, M. Yamanishi, M. Otsubo, Y. Ohashi and J. Negishi provided medical care and contributed to the preparation of the manuscript. H. Terada and T. Fuchigami drafted the manuscript. T. Fuchigami designed the manuscript outline and provided conceptual advice. I. Morioka supervised the entire study. All authors read and approved the final manuscript.

\section{Data Availability}

The authors declare that data supporting the findings of this study are available within the article.

\section{References}

1. Rosenfield AT, Glickman MG, Taylor KJ, Crade M, Hodson J. Acute focal bacterial nephritis (acute lobar nephronia). Radiology. 1979;132(3):553-561.

2. Cox SM, Cunningham FG. Acute focal pyelonephritis (lobar nephronia) complicating pregnancy. Obstet Gynecol. 1988;71(3 Pt 2):510-511.

3. Cheng CH, Tsau YK, Lin TY. Effective duration of antimicrobial therapy for the treatment of acute lobar nephronia. Pediatrics. 2006;117(1):e84-89.

4. Saito K, Fuchigami T, Hasegawa M, Kawamura Y, Hashimoto K, Fijita Y, Inamo Y, et al. Clinical features of acute focal bacterial nephritis in children. Int J Clin Pediatr. 2013;2(2):68-73.

5. Kline MW, Kaplan SL, Baker CJ. Acute focal bacterial nephritis: diverse clinical presentations in pediatric patients. Pediatr Infect Dis J. 1988;7(5):346-349.

6. Kometani H, Kawatani M, Ohta G, Okazaki S, Ogura $\mathrm{K}$, Yasutomi M, Tanizawa A, et al. Marked elevation of interleukin-6 in mild encephalopathy with a reversible splenial lesion (MERS) associated with acute focal bacterial nephritis caused by Enterococcus faecalis. Brain Dev.
2014;36(6):551-553

7. Kasuga Y, Fuchigami T, Fukuda A, Takahashi S, Murai $\mathrm{T}$, Yonezawa R, Miyashita M, et al. Acute focal bacterial nephritis associated with central nervous system manifestations: a report of 2 cases and review of the literature. Pediatr Emerg Care. 2017;33(6):418-421.

8. Tada H, Takanashi J, Barkovich AJ, Oba H, Maeda M, Tsukahara H, Suzuki M, et al. Clinically mild encephalitis/encephalopathy with a reversible splenial lesion. Neurology. 2004;63(10):1854-1858.

9. Takanashi J, Imamura A, Hayakawa F, Terada H. Differences in the time course of splenial and white matter lesions in clinically mild encephalitis/encephalopathy with a reversible splenial lesion (MERS). J Neurol Sci. 2010;292(1-2):24-27.

10. Hoshino A, Saitoh M, Oka A, Okumura A, Kubota M, Saito Y, Takanashi J, et al. Epidemiology of acute encephalopathy in Japan, with emphasis on the association of viruses and syndromes. Brain Dev. 2012;34(5):337-343.

11. Takanashi J. Two newly proposed infectious encephalitis/ encephalopathy syndromes. Brain Dev. 2009;31(7):521528.

12. Okamoto T, Sato Y, Yamazaki T, Hayashi A. Clinically mild encephalitis/encephalopathy with a reversible splenial lesion associated with febrile urinary tract infection. Eur J Pediatr. 2014;173(4):533-536.

13. Takanashi J, Tada H, Maeda M, Suzuki M, Terada H, Barkovich AJ. Encephalopathy with a reversible splenial lesion is associated with hyponatremia. Brain Dev. 2009;31(3):217-220.

14. Soulen MC, Fishman EK, Goldman SM, Gatewood OM. Bacterial renal infection: role of CT. Radiology. 1989;171(3):703-707.

15. Jantausch BA, Rifai N, Getson P, Akram S, Majd M, Wiedermann BL. Urinary N-acetyl-beta-glucosaminidase and beta-2-microglobulin in the diagnosis of urinary tract infection in febrile infants. Pediatr Infect Dis J. 1994;13(4):294-299.

16. Sekine H, Kawasaki Y, Ohara S, Suyama K, Hosoya M. Focal bacterial nephritis without pyuria in a boy presenting with high urinary beta2-MG and NAG levels. Fukushima J Med Sci. 2014;60(1):91-94. 